\title{
The Impact of Stochastic Primordial Magnetic Fields on the Scalar Contribution to Cosmic Microwave Background Anisotropies
}

\author{
Fabio Finell:* \\ INAF/IASF-BO, Istituto di Astrofisica Spaziale e Fisica Cosmica di Bologna \\ via Gobetti 101, I-40129 Bologna - Italy \\ INAF/OAB, Osservatorio Astronomico di Bologna, via Ranzani 1, I-40127 Bologna - Italy and \\ INFN, Sezione di Bologna, Via Irnerio 46, I-40126 Bologna, Italy \\ Francesco Pacit \\ Dipartimento di Astronomia, \\ Università degli Studi di Bologna, \\ via Ranzani, 1 - I-40127 Bologna - Italy \\ INAF/IASF-BO, Istituto di Astrofisica Spaziale e Fisica Cosmica di Bologna \\ via Gobetti 101, I-40129 Bologna - Italy and \\ INFN, Sezione di Bologna, Via Irnerio 46, I-40126 Bologna, Italy \\ Daniela Paolett团 \\ Dipartimento di Fisica, Università degli Studi di Ferrara, \\ via Saragat, 1 - I-44100 Ferrara - Italy \\ INAF/IASF-BO, Istituto di Astrofisica Spaziale e Fisica Cosmica di Bologna \\ via Gobetti 101, I-40129 Bologna - Italy and \\ INFN, Sezione di Bologna, Via Irnerio 46, I-40126 Bologna, Italy
}

\begin{abstract}
We study the impact of a stochastic background of primordial magnetic fields on the scalar contribution of CMB anisotropies and on the matter power spectrum. We give the correct initial conditions for cosmological perturbations and the exact expressions for the energy density and Lorentz force associated to the stochastic background of primordial magnetic fields, given a power-law for their spectra cut at a damping scale. The dependence of the CMB temperature and polarization spectra on the relevant parameters of the primordial magnetic fields is illustrated.

PACS numbers: $98.80 . \mathrm{Cq}$
\end{abstract}

\section{INTRODUCTION}

Large scale magnetic fields are almost everywhere in the universe, from galaxies up to those present in galaxy clusters and in the intercluster medium [1]. The origin of these magnetic fields depends on the size of the objects and may become mysterious for the largest ones. The dynamo mechanism provides a mechanism to explain the observed magnetic field associated to galaxies, whereas those associated to clusters may be generated by gravitational compression starting from an initial seed.

The requirement of an initial seed for magnetic fields observed in galaxies and galaxy clusters leads directly to question the existence of primordial magnetic fields in the early universe. Cosmology described by an homogeneous and isotropic expanding metric neither supports a uniform magnetic field nor a gravitational amplification of gauge fields because of conformal invariance; the generation of large scale magnetic fields has therefore generated a lot of interest. A stochastic background (SB) of primor-

\footnotetext{
*Electronic address: finelli@iasfbo.inaf.it

${ }^{\dagger}$ Electronic address: paci@iasfbo.inaf.it

${ }^{\ddagger}$ Electronic address: paoletti@iasfbo.inaf.it
}

dial magnetic fields (PMF) can provide the initial seeds for the large-scale magnetic fields observed and can leave imprints on different observables, as the CMB pattern of temperature and polarization anisotropies [2, 3] and the matter power spectrum.

A SB of PMF carries zero energy and pressure at homogeneous level in a Robertson-Walker metric. It carries however perturbations, of any kind, i.e. scalar, vector and tensor, and it is usually studied in a quasi-linear approximation, i.e. its EMT - quadratic in the magnetic field amplitude - is considered at the same footing as first order terms in a perturbative series expansion. Vector [4, 5] and tensor [4, 6, 7] metric perturbations sourced by a PMF SB have been object of several investigations; beyond the technical simplicity of vector and tensor over scalar, a perfect fluid cannot support vector and tensor perturbations at linear order and therefore represent a key prediction of a PMF SB. We know however that temperature and polarization anisotropies sourced by scalar fluctuations with adiabatic initial conditions are a good fit to the whole set of observations; it is therefore crucial to investigate how a PMF SB can modify these scalar fluctuations. Analytic [8] and numerical [9, 10, 11] works in this direction have already been made. However a detailed analysis which takes into account the Lorentz force on baryons, a careful treatment of initial conditions 
and an accurate treatment of the Fourier spectra of PMF energy-momentum tensor is still lacking. As is clear in the following, our work address carefully both these issues.

The goal of this paper is to investigate the impact of a stochastic background (SB) of primordial magnetic fields (PMF) on scalar cosmological perturbations and in particular on $\mathrm{CMB}$ temperature anisotropies and matter power spectrum. Our paper is organized as follows. In Section II we review how to add a fully inhomogenous SB of PMF treated in the one-fluid plasma description [2] to the Einstein-Boltzmann system of equations. In Sections III and IV we review the baryons evolution and we give the initial conditions for cosmological perturbations in a form suitable to be plugged in most of the EinsteinBotzmann codes. In Section $\mathrm{V}$ we give the PMF energy density and Lorentz force power spectra and compare our results with the ones given in the literature. In Sections VI-VIII we show the results obtained by our modification of the Einstein-Boltzmann code CAMB [18] for cosmological scalar perturbations, CMB spectrum of temperature and polarization, matter power spectrum, respectively. In the Appendix we show the detailed calculations for the convolution integrals leading to the energy density and Lorentz force, starting from a power-law spectrum sharply cut at a given scale for the PMF.

\section{STOCHASTIC MAGNETIC FIELDS AND COSMOLOGICAL SCALAR PERTURBATIONS}

We model a SB of PMFs as a fully inhomogenous component, considering $B^{2}$ at the same level of metric and density fluctuations in a perturbative expansion *. Although a SB of PMFs carries no energy at the homogeneous level, it affects scalar cosmological perturbations in three different ways. First, inhomogeneous PMFs carry energy density and pressure and therefore gravitate at the level of perturbations. Second, inhomogeneous PMFs have anisotropic stress - differently from perfect fluids which adds to the photon and neutrino ones, with the caveat that the photon anisotropic stress is negligible before the decoupling epoch. Last, but not least, the induced Lorentz force acting on baryons, affects also photons during the tight coupling regime.

Since the EMT of PMF at homogeneous level is zero, at linear order PMFs evolve like a stiff source and therefore it is possible to discard all the back reactions of the fluid or gravity onto the SB of PMF. Before the decoupling epoch the electric conductivity of the primordial

\footnotetext{
* Note that in such a way we do not take into account the modification of the sound speed of baryons induced by PMFs, pionereed in [12], since it would be technically of second order in the equations of motion. However, since the baryons speed of sound goes rapidly to zero in the matter dominated era, this effect, leading to a shift in the Doppler peaks, may be anyway important.
}

plasma is very large, therefore it is possible at the first order to consider the infinite conductivity limit. In this limit the induced electric field is zero. Within the infinite conductivity limit the SB of PMF time evolution simply reduces to : $\mathbf{B}(\mathbf{x}, \tau)=\mathbf{B}(\mathbf{x}) / a(\tau)^{2} .^{\dagger}$

The evolution of the metric perturbations in the presence of PMF is governed by the Einstein equations:

$$
G_{\mu \nu}=8 \pi\left(T_{\mu \nu}+\tau_{\mu \nu}^{\mathrm{PMF}}\right)
$$

In the approximation in which the induced electric field is vanishing (i.e. the infinite conductivity limit) the energy momentum tensor of the electromagnetic field becomes:

$$
\begin{aligned}
\tau_{0}^{0 \mathrm{PMF}} & =-\rho_{B}=-\frac{|\mathbf{B}(\mathbf{x})|^{2}}{8 \pi a^{4}} \\
\tau_{i}^{0 \mathrm{PMF}} & =0 \\
\tau_{j}^{i \mathrm{PMF}} & =\frac{1}{4 \pi a^{4}}\left(\frac{|\mathbf{B}(\mathbf{x})|^{2}}{2} \delta_{j}^{i}-B_{j}(\mathbf{x}) B^{i}(\mathbf{x})\right) .
\end{aligned}
$$

In the Fourier space ${ }^{\ddagger}$ the Einstein equations with the contribution of PMF in the synchronous gauge are:

$$
\begin{aligned}
k^{2} \eta-\frac{1}{2} \mathcal{H} \dot{h}= & 4 \pi G a^{2}\left(\Sigma_{n} \rho_{n} \delta_{n}+\rho_{B}\right), \\
k^{2} \dot{\eta}= & 4 \pi G a^{2} \Sigma_{n}\left(\rho_{n}+P_{n}\right) \theta_{n}, \\
\ddot{h}+2 \mathcal{H} \dot{h}-2 k^{2} \eta= & -8 \pi G a^{2}\left(\Sigma_{n} c_{s n}^{2} \rho_{n} \delta_{n}\right. \\
& \left.+\frac{\rho_{B}}{3}\right), \\
\ddot{h}+6 \ddot{\eta}+2 \mathcal{H}(\dot{h}+6 \dot{\eta})-2 k^{2} \eta= & -24 \pi G a^{2} \times \\
& {\left[\Sigma_{n}\left(\rho_{n}+P_{n}\right) \sigma_{n}+\sigma_{B}(\hat{\phi}]\right) }
\end{aligned}
$$

where by $n$ we mean the number of components, i.e. baryons, cold dark matter (CDM), photons and neutrinos. The conservation of the PMF EMT $-\nabla_{\mu} \tau_{\nu}^{\mu \mathrm{PMF}}=0$ - simply reduces to :

$$
\sigma_{B}=\frac{\rho_{B}}{3}+L
$$

where $\sigma_{B}$ represents the PMFs anisotropic stress and $L$ the Lorentz force. The energy density of PMF evolves like radiation: $\rho_{B}(\mathbf{x}, \tau)=\rho_{B}\left(\mathbf{x}, \tau_{0}\right) / a(\tau)^{4}$.

\section{BARYONS EVOLUTION}

The presence of PMFs in a plasma which contains charged particles induces a Lorentz force on these particles, that, in the primordial plasma, are baryons. The

$\dagger$ We choose the standard convention in which at present time $t_{0}$, $a\left(t_{0}\right)=1$.

$\ddagger$ As Fourier transform and its inverse, we use - in agreement with [13] -:

$Y(\vec{k}, \tau)=\int \frac{d^{3} x}{(2 \pi)^{3}} e^{-i \vec{k} \cdot \vec{x}} Y(\vec{x}, \tau), \quad Y(\vec{x}, \tau)=\int d^{3} k e^{i \vec{k} \cdot \vec{x}} Y(\vec{k}, \tau)$.

where $Y$ is a generic function. 
general expression for the Lorentz force is [8]:

$$
L_{i}\left(x, \tau_{0}\right)=\frac{1}{4 \pi}\left[B_{j}(\mathbf{x}) \nabla_{j} B_{i}(\mathbf{x})-\frac{1}{2} \nabla_{i} B^{2}(\mathbf{x})\right],
$$

where $\mathbf{L}(x, \tau)=\frac{\mathbf{L}\left(x, \tau_{0}\right)}{a^{4}}$.

We are interested only in the scalar perturbations and the scalar part of the Lorentz force defined as $\nabla^{2} L^{(S)} \equiv \nabla_{i} L_{i}$ is therefore:

$$
\nabla^{2} L^{(S)}=\frac{1}{4 \pi}\left[\left(\nabla_{i} B_{j}(\mathbf{x})\right) \nabla_{j} B_{i}(\mathbf{x})-\frac{1}{2} \nabla^{2} B^{2}(\mathbf{x})\right] .
$$

In the presence of an electromagnetic source the conservation equations of the baryon component of the primordial fluid becomes:

$$
\nabla_{\mu} \delta T^{\mu \nu \text { baryons }} \propto F^{\mu \nu} J_{\mu}
$$

where $J_{\mu}$ is the quadrivector of the density current and $F^{\mu \nu}$ is the Maxwell tensor. The primordial plasma can be considered globally neutral, this leads to $J_{0}=0$ and therefore to the fact that the energy conservation of baryons is not modified by the presence of the Lorentz term. The Euler equation for baryons in instead affected by the Lorentz force and the scalar part is therefore [2]:

$$
\dot{\theta}_{b}=-\mathcal{H} \theta_{b}+k^{2} c_{s b}^{2} \delta_{b}-k^{2} \frac{L}{\rho_{b}} .
$$

Now we study how the tight-coupling regime is modified by the presence of a SB of PMF [14]. The Euler equation for photons during the tight-coupling regime is:

$$
\dot{\theta}_{\gamma}=k^{2}\left(\frac{\delta_{\gamma}}{4}-\sigma_{\gamma}\right)+a n_{e} \sigma_{T}\left(\theta_{b}-\theta_{\gamma}\right)
$$

Combining the photons and baryons equations gives:

$\dot{\theta}_{b}=\frac{-\mathcal{H} \theta_{b}+c_{s}^{2} k^{2} \delta_{b}+k^{2} R\left(\frac{\delta_{\gamma}}{4}-\sigma_{\gamma}\right)+R\left(\dot{\theta}_{\gamma}-\dot{\theta}_{b}\right)-\frac{k^{2} L}{\rho_{b}}}{(1+R)}$

with:

$$
\begin{aligned}
\dot{\theta}_{b}-\dot{\theta}_{\gamma}= & \frac{2 R}{(1+R)} \mathcal{H}\left(\theta_{b}-\theta_{\gamma}\right)+\frac{\tau}{(1+R)}\left(-\frac{\ddot{a}}{a} \theta_{b}+\right. \\
& \left.-\frac{\mathcal{H} k^{2}}{2} \delta_{\gamma}+k^{2}\left(c_{s}^{2} \dot{\delta}_{b}-\frac{\dot{\delta}_{\gamma}}{4}\right)+\mathcal{H} k^{2} \frac{L}{\rho_{b}}\right),
\end{aligned}
$$

The photon Euler equation in tight coupling regime instead is:

$$
\begin{aligned}
\dot{\theta}_{\gamma}= & -R^{-1}\left(\dot{\theta}_{b}+\mathcal{H} \theta_{b}-c_{s}^{2} k^{2} \delta_{b}+k^{2} \frac{L}{\rho_{b}}\right) \\
& +k^{2}\left(\frac{\delta_{\gamma}}{4}-\sigma_{\gamma}\right)
\end{aligned}
$$

We note that there is a term depending on the Lorentz force which disappears when the tight coupling ends, leaving the normal Euler equation for the photon velocity.

\section{INITIAL CONDITIONS}

In order to study the effect of a PMF SB on scalar cosmological perturbations, the initial conditions for the latter deep in the radiation era are required (see [2] for the results in the longitudinal gauge). The magnetized adiabatic mode initial conditions in the synchronous gauge are given by [15]:

$$
\begin{aligned}
h= & C_{1}(k \tau)^{2} \\
\eta= & 2 C_{1}-\frac{5+4 R_{\nu}}{6\left(15+4 R_{\nu}\right)} C_{1}(k \tau)^{2}+ \\
& -\left[\frac{\Omega_{B}\left(1-R_{\nu}\right)}{6\left(15+4 R_{\nu}\right)}+\frac{L_{B}}{2\left(15+4 R_{\nu}\right)}\right](k \tau)^{2} \\
\delta_{\gamma}= & -\Omega_{B}-\frac{2}{3} C_{1}(k \tau)^{2}+\left[\frac{\Omega_{B}}{6}+\frac{L_{B}}{2\left(1-R_{\nu}\right)}\right](k \tau)^{2} \\
\delta_{\nu}= & -\Omega_{B}-\frac{2}{3} C_{1}(k \tau)^{2}-\left[\frac{\Omega_{B}\left(1-R_{\nu}\right)}{6 R_{\nu}} \frac{L_{\nu}}{2 R_{\nu}}\right](k \tau)^{2} \\
\delta_{b}= & -\frac{3}{4} \Omega_{B}-\frac{C_{1}}{2}(k \tau)^{2}+\left[\frac{\Omega_{B}}{8}+\frac{3 L_{B}}{8\left(1-R_{\nu}\right)}\right](k \tau)^{2} \\
\delta_{c}= & -\frac{C_{1}}{2}(k \tau)^{2} \\
\theta_{\gamma}= & -\frac{C_{1}}{18} k^{4} \tau^{3}+\left[-\frac{\Omega_{B}}{4}-\frac{3}{4} \frac{L_{B}}{\left(1-R_{\nu}\right)}\right] k^{2} \tau \\
& +k\left[\frac{\Omega_{B}}{72}+\frac{L_{B}}{24\left(1-R_{\nu}\right)}\right](k \tau)^{3} \\
\theta_{b}= & \theta_{\gamma} \\
\theta_{c}= & 0 \\
\theta_{\nu}= & -\frac{\left(23+4 R_{\nu}\right)}{18\left(15+4 R_{\nu}\right)} C_{1} k^{4} \tau^{3}+\left[\frac{\Omega_{B}\left(1-R_{\nu}\right)}{4 R_{\nu}}+\frac{3}{4} \frac{L_{B}}{R_{\nu}}\right] k^{2} \tau \\
& -\left[\frac{\left(1-R_{\nu}\right)\left(27+4 R_{\nu}\right) \Omega_{B}}{72 R_{\nu}\left(15+4 R_{\nu}\right)}+\frac{\left(27+4 R_{\nu}\right) L_{B}}{24 R_{\nu}\left(15+4 R_{\nu}\right)}\right] k^{4} \tau^{3} \\
\sigma_{\nu}= & \frac{4 C_{1}}{3\left(15+4 R_{\nu}\right)}(k \tau)^{2}-\frac{\Omega_{B}}{4 R_{\nu}}-\frac{3}{4} \frac{L_{B}}{R_{\nu}} \\
& +\left[\frac{\left(1-R_{\nu}\right)}{R_{\nu}\left(15+4 R_{\nu}\right)} \frac{\Omega_{B}}{2}+\frac{3}{2} \frac{L_{B}}{R_{\nu}\left(15+4 R_{\nu}\right)}\right](k \tau)^{2},(14) \\
& R^{2}(14)
\end{aligned}
$$

where $R_{\nu}=\rho_{\nu} /\left(\rho_{\nu}+\rho_{\gamma}\right)$ and $C_{1}$ is the constant which characterize the regular growing adiabatic mode as given in [13]. We have checked that the result reported in [11] and ours [15] agree.

Note how the presence of a SB of PMFs induces a new independent mode in matter and metric perturbations, i.e. the fully magnetic mode. This new independent mode is the particular solution of the inhomogeneous system of the Einstein-Botzmann differential equations: the SB of PMF treated as a stiff source acts indeed as a force term in the system of linear differential equations. Whereas the sum of the fully magnetic mode with the curvature one can be with any correlation as for an isocurvature mode, the nature of the fully magnetic mode - and therefore its effect - is different: the isocurvature modes are solutions of the homogeneous system (in which all the species have both background and perturbations), 
whereas the fully magnetic one is the solution of the inhomogeneous system sourced by a fully inhomogeneous component.

It is interesting to note the magnetic contribution drops from the metric perturbation at leading order, although is actually larger than the adiabatic solution for photons, neutrinos and baryons (the latter being tightly coupled to photons deep in the radiation era). This is due to a compensation which nullifies the sum of the leading contributions (in the long-wavelength expansion) in the single species energy densities and therefore in the metric perturbations. A similar compensation exists for a network of topological defects, which does not carry a background energy-momentum tensor as the PMF SB studied here $\S$.

\section{MAGNETIC FIELD POWER SPECTRA}

Power spectra for the amplitude and the EMT of SB of PMF have been subject of several investigation [4, 6, 8, 9]. We shall work in the Fourier space according to Eq. (5). We shall consider PMFs with a power law power spectrum, which therefore are characterize by two parameters: an amplitude $A$ and a spectral index $n_{B}$. PMFs are suppressed by radiation viscosity on small scales: we approximate this damping by introducing an ultraviolet cut-off in the power spectrum at the (damping) scale $k_{D}$.

The two-point correlation function for a statistically homogeneous and isotropic field is

$$
\begin{aligned}
\left\langle\overrightarrow{B_{i}^{*}}(\vec{k}) \overrightarrow{B_{j}}\left(\overrightarrow{k^{\prime}}\right)\right\rangle=\delta^{3}\left(\vec{k}-\overrightarrow{k^{\prime}}\right) \quad\left[\left(\delta_{i j}-\hat{k}_{i} \hat{k}_{j}\right) \frac{P_{B}(k)}{2}+\right. & \left.\epsilon_{i j l} \frac{k_{l}}{P_{H}}(k)\right]
\end{aligned}
$$

$\S$ Note however that a network of topologickl defects ]does not scale with radiation and interacts only gravitationally with the rest of where $\epsilon_{i j l}$ is the totally antisymmetric tensor, $P_{B}$ and $P_{H}$ are the non-helical and helical part of the spectrum for the $\mathbf{B}$ amplitude, respectively. Scalar cosmological perturbations only couple to the non-helical part of the spectrum and we shall therefore consider only $P_{B}$ in the following.

\section{A. Magnetic Energy Density}

As is clear from Eqs. (1-3), the EMT for PMF is quadratic in the field amplitude. The PMF energy density spectrum is [9]:

$$
\left|\rho_{B}(k)\right|^{2}=\frac{1}{128 \pi^{2} a^{8}} \int d^{3} p P_{B}(\vec{p}) P_{B}(|\vec{k}-\vec{p}|)\left(1+\mu^{2}\right),
$$

where $\mu=\frac{\vec{p}(\vec{k}-\vec{p})}{p|\vec{k}-\vec{p}|}=\frac{k \cos \theta-p}{\sqrt{\left(k^{2}+p^{2}-2 k p \cos \theta\right)}}$. As for the twopoint function in the coincidence limit, for several physical spectral indexes and PMF configurations such convolution is not finite. There are in general problems both on large and short scales. Since the spectrum of the components of PMF EMT are relevant for the final impact on cosmological perturbations and $\mathrm{CMB}$ anisotropies, it is better to address this point in much more detail with respect to what is present in literature.

matter, i.e. a Lorentz term is absent.

The usual choice in the literature is to modify the scalar part two-point function of Eq. (15) for zero helicity as [8]:

$$
\left\langle\overrightarrow{B_{i}^{*}}(\vec{k}) \vec{B}_{j}\left(\overrightarrow{k^{\prime}}\right)\right\rangle= \begin{cases}\delta^{3}\left(\vec{k}-\overrightarrow{k^{\prime}}\right)\left(\delta_{i j}-\hat{k}_{i} \hat{k}_{j}\right) \frac{P_{B}(k)}{2} & \text { for } \quad k<k_{D} \\ 0 & \text { for } k>k_{D}\end{cases}
$$

with

$$
P_{B}(k)=A\left(\frac{k}{k_{*}}\right)^{n_{B}}
$$

where $k_{*}$ is a reference scale. With such choice the twopoint function in the coincident limit (the mean square of the magnetic field) is:

$$
\begin{aligned}
\left\langle B^{2}(x)\right\rangle & =\int_{k<k_{D}} d^{3} k P_{B}(k) \\
& =\frac{4 \pi A}{n_{B}+3} \frac{k_{D}^{n_{B}+3}}{k_{*}^{n_{B}}} .
\end{aligned}
$$

It is also usual in the literature to give the amplitude of $B$ at a given smearing scale $k_{S}$ by imposing a Gaussian filter :

$$
\begin{aligned}
\left\langle B^{2}(x)\right\rangle_{k_{S}} & =\int d^{3} k P_{B}(k) e^{-k^{2} / k_{S}^{2}} \\
& =2 \pi A \frac{k_{S}^{n_{B}+3}}{k_{*}^{n_{B}}} \Gamma\left(\frac{n_{B}+3}{2}\right) .
\end{aligned}
$$




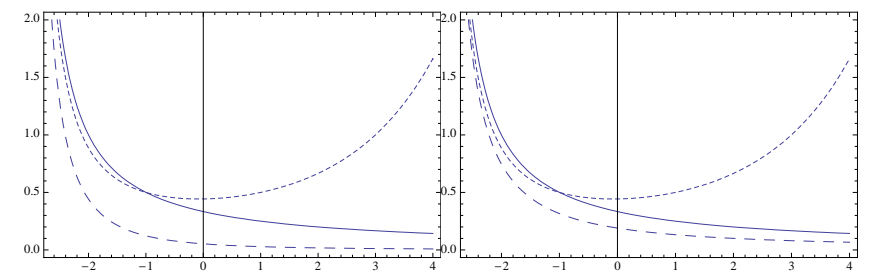

FIG. 1: Plots of the different ways of computing the magnetic power spectra (in units of $4 \pi A k_{D}^{n_{B}+3} / k_{*}^{n_{B}}$ ) versus $n_{B}$ for $k_{S}=$ $k_{D} / 2$ (left) and $k_{S}=k_{D}$ (right). $\left\langle B^{2}\right\rangle,\left\langle B^{2}\right\rangle_{k_{S}},\left\langle B^{2}\right\rangle_{\text {cut }, k_{S}}$ are represented by solid, dotted and dashed lines, respectively.

By smearing the magnetic power spectrum and integrating for $k<k_{D}$, one gets:

$$
\begin{aligned}
\left\langle B^{2}(x)\right\rangle_{k_{S}}^{\text {cut }}= & \int_{k<k_{D}} d^{3} k P_{B}(k) e^{-k^{2} / k_{S}^{2}} \\
= & 2 \pi A \frac{k_{D}^{n_{B}+3}}{k_{*}^{n_{B}}}\left[\Gamma\left(\frac{n_{B}+3}{2}\right)\right. \\
& \left.-\Gamma\left(\frac{n_{B}+3}{2}, \frac{k_{D}^{2}}{k_{S}^{2}}\right)\right],
\end{aligned}
$$

where the incomplete Gamma function $\Gamma(\ldots, \ldots)$ [17 has been introduced. Note how $n_{B}>-3$ in order to prevent infrared divergencies either in the mean square field or the amplitude of the field smeared at a given scale. In the following by $\left\langle B^{2}\right\rangle$ we mean the value given by Eq. (18). Fig. (1) shows how $\left\langle B^{2}(x)\right\rangle_{k_{S}}$ may be much larger than $\left\langle B^{2}\right\rangle$ for $n_{B}>0$.

The exact result for the Fourier convolution leading to the magnetic energy density Fourier square amplitude is one of the new main results of this paper. The convolution involves a double integral, one in the angle between $k$ and $p$ and one in the modulus of $p$. The integral in the angle, often omitted in the literature, is the reason for having the result for $\left|\rho_{B}(k)\right|^{2}$ non vanishing only for $k<2 k_{D}$. The detailed calculations for the energy density convolutions are given in Appendix A for several values of $n_{B}$. The generic behaviour for $k<<k_{D}$ and $n_{B}>-3 / 2$ is white noise with amplitude

$$
\left|\rho_{B}(k)\right|^{2} \simeq \frac{A^{2} k_{D}^{2 n+3}}{16 \pi k_{*}^{2 n}\left(3+2 n_{B}\right)}
$$

and then goes to zero for $k=2 k_{D}$, which is a result obtained by performing correctly the integral. The pole for $n_{B}=-3 / 2$ in Eq. (21) is replaced by a logarithmic diveregence in $k$ in the exact result; for $n_{B}<-3 / 2$ the spectrum is no more white noise for $k<<k_{D}$. Fig. (2) shows the dependence of $k^{3}\left|\rho_{B}(k)\right|^{2}$ on $n_{B}$ at fixed $\left\langle B^{2}\right\rangle$.

Our result are different from the one reported in the literature [8], which is

$$
\left|\rho_{B}(k)\right|_{K R}^{2}=\frac{3 A^{2} k_{D}^{2 n_{B}+3}}{64 \pi k_{*}^{2 n_{B}}\left(3+2 n_{B}\right)}\left[1+\frac{n_{B}}{n_{B}+3}\left(\frac{k}{k_{D}}\right)^{2 n_{B}+3}\right],
$$

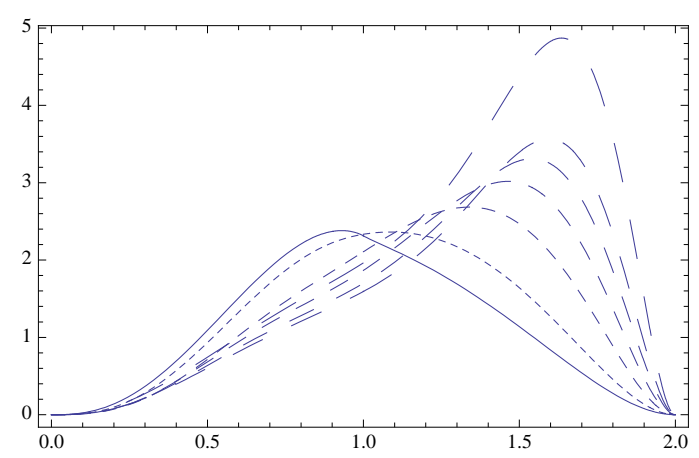

FIG. 2: Plot of magnetic energy density power spectrum $k^{3}\left|\rho_{B}(k)\right|^{2}$ in units of $\left\langle B^{2}\right\rangle^{2} /\left(1024 \pi^{3}\right)$ versus $k / k_{D}$ for different $n_{B}$ for fixed $\left\langle B^{2}\right\rangle$. The different lines are for $n_{B}=$ $-3 / 2,-1,0,1,2,3,4$ ranging from the solid to the longest dashed.

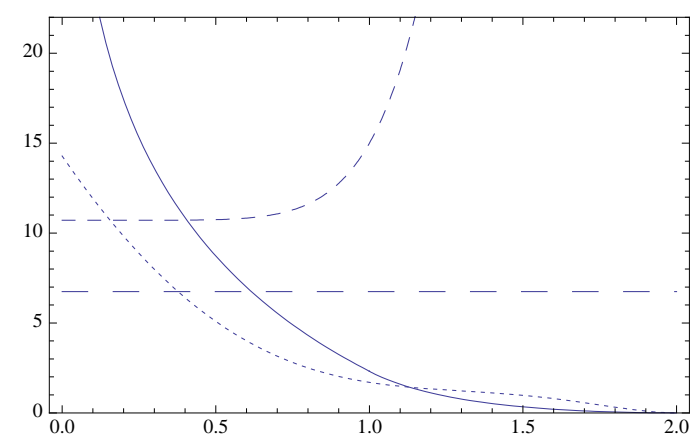

FIG. 3: Comparison of magnetic energy density convolution $k_{D}^{3}\left|\rho_{B}(k)\right|^{2}$ obtained in this paper (dotted, solid) in units of $\left\langle B^{2}\right\rangle^{2} /\left(1024 \pi^{3}\right)$ and the one in Eq. (22) (dashed, longdashed) versus $k / k_{D}$ for $n_{B}=2,-3 / 2$ with fixed $\left\langle B^{2}\right\rangle$.

and is not limited in $k$. In Fig. (3) we show the difference between the literature result [8] and our result for $n_{B}=$ $2,-3 / 2$.

\section{B. Lorentz Force}

As is clear from previous sections, we also need the Lorentz force

$$
|L(k)|^{2}=\frac{1}{128 \pi^{2} a^{8}} \int d^{3} p \quad P_{B}(p) P_{B}(|\mathbf{k}-\mathbf{p}|)\left[1+\mu^{2}+4 \gamma \beta(\gamma \beta-\mu)\right],
$$


and the magnetic anisotropic stress

$$
\left|\sigma_{B}(k)\right|^{2}=\frac{1}{288 \pi^{2} a^{8}} \int d^{3} p P_{B}(p) P_{B}(|\mathbf{k}-\mathbf{p}|)\left[9\left(1-\gamma^{2}\right)\left(1-\beta^{2}\right)-6\left(1+\gamma \mu \beta-\gamma^{2}-\beta^{2}\right)\left(1+\mu^{2}\right)\right],
$$

where $\gamma=\hat{k} \cdot \hat{p}, \beta=\vec{k} \cdot(\vec{k}-\vec{p}) /(k|\vec{k}-\vec{p}|)$ and $\mu=\vec{p} \cdot(\vec{k}-\vec{p}) /(p|\vec{k}-\vec{p}|)$.

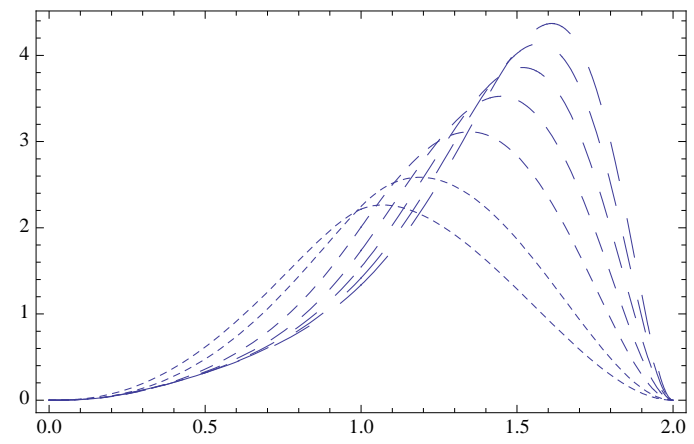

FIG. 4: Plot of the Lorentz force power spectrum $k^{3}|L(k)|^{2}$ in units of $\left\langle B^{2}\right\rangle^{2} /\left(1024 \pi^{3}\right)$ versus $k / k_{D}$ for different $n_{B}$ for fixed $\left\langle B^{2}\right\rangle$. The different lines are for $n_{B}=-3 / 2,-1,0,1,2,3$ ranging from the solid to the longest dashed.

We decide to compute the spectrum of the Lorentz force and obtain the anisotropic stress by Eq. (7). The exact computation for the Lorentz force power spectrum is given in Appendix $\mathrm{B}$ for several values of $n_{B}$. A term $-\rho_{B}$ can be easily identified in Eq. (9); since we know from the exact computation that the integral of $P_{B}(p) P_{B}(|\mathbf{k}-\mathbf{p}|)\left(1+\mu^{2}\right)$ is larger than the remaining piece in Eq. (23) we chose the the signs for $\rho_{B}(k)$ and $L(k)$ as opposite.

Fig. (4) shows the dependence of $k^{3}|L(k)|^{2}$ on $n_{B}$ at fixed $\left\langle B^{2}\right\rangle$. Fig. (5) compares the approximation $L(k) \simeq$ $-\rho_{B}(k)$ suggested in Ref. [8] with the exact calculation. As can be checked in Appendix B, our exact calculations for the values of $n_{B}$ studied here show that

$$
|L(k)|^{2} \simeq \frac{11}{15}\left|\rho_{B}(k)\right|^{2} \quad \text { for } \quad k<<k_{D} .
$$

\section{RESULTS FOR COSMOLOGICAL PERTURBATIONS}

In order to study the effects of a SB of PMFs on CMB anisotropies and matter power spectrum we modified the CAMB Einstein-Boltzmann code [18] (June 2006 version) by introducing the PMF contribution in the Einstein equations, in the evolution equation for baryons and initial conditions, along Eqs. 6[26]14).

We note that implementing the baryons evolution as from Eq. (26), the MHD approximation in a globally neutral plasma is used up to the present time: this makes the Lorentz term non-vanishing up to the present time. Although the term $L / \rho_{b}$ in Eq. (26) decreases with time, its

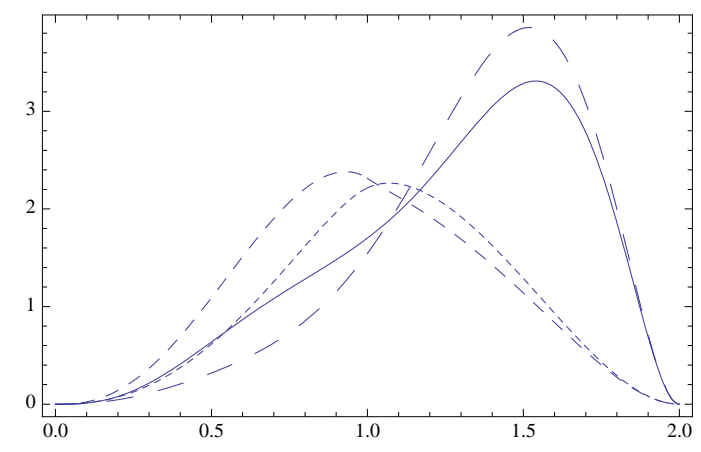

FIG. 5: Comparison of the magnetic energy density and Lorentz force power spectra versus $k / k_{D}$ for fixed $\left\langle B^{2}\right\rangle$. The solid (medium dashed) and long-dashed (short-dashed) lines are respectively for $k^{3}\left|\rho_{B}(k)\right|^{2}$ and $k^{3}|L(k)|^{2}$ for $n_{B}=2$ $\left(n_{B}=-3 / 2\right)$.

effect on the baryon velocity is crucial. Much later than the larger between the decoupling time and the time at which sound speed of baryons is effectively zero, baryons velocity can be approximated as:

$$
\theta_{b}^{\text {late }} \simeq-k^{2}\left(\frac{L a}{\rho_{b}}\right) \frac{\tau}{a}
$$

during the matter dominated era. Our modified EinsteinBoltzmann code reproduces correctly this asymptotic regime for different wavelengths, as can be seen by Fig. (6). The corresponding effects on the density contrasts for the same wavelengths are shown in Fig. (7). In Fig. (8) the effects due to the pure magnetic mode and due to the correlation with the adiabatic mode are shown. Fig. (9) displays the importance of the Lorentz term compared to the purely gravitational effect.

\section{RESULTS FOR CMB TEMPERATURE AND POLARIZATION POWER SPECTRA}

In this section we show the results on the CMB temperature and polarization pattern obtained by our modifications of the CAMB code. Fig. (10) shows the various contributions to the total $\mathrm{CMB}$ temperature and polarization angular power spectra from the pure magnetic mode and its correlation with the adiabatic mode. Fig. (11) shows the dependence of the total temperature power spectrum on the spectral index $n_{B}$.

As is clear from the previous section, the Lorentz force of a fully correlated magnetic contribution decreases the 

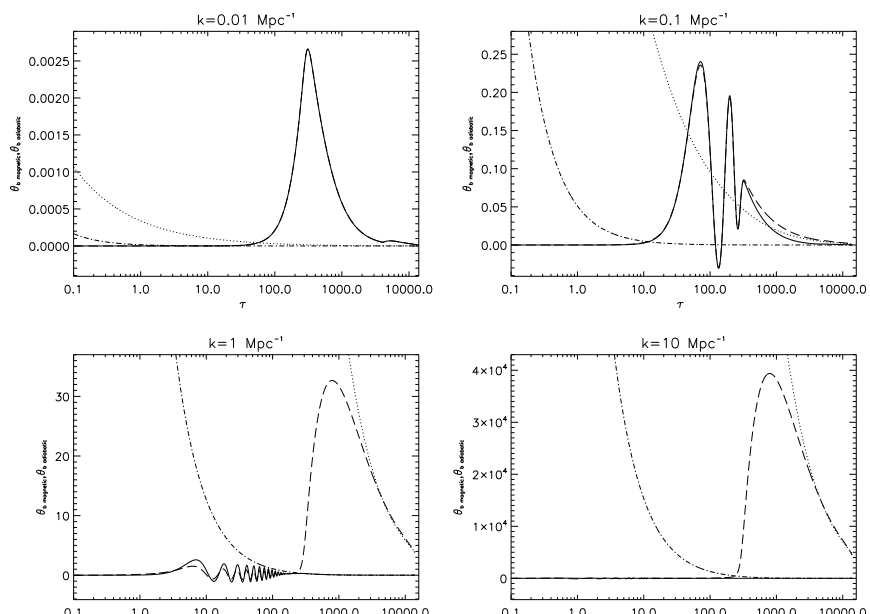

FIG. 6: Evolution of baryons velocity for 4 different wavenumbers with (dashed) and without (solid) PMF. $k^{2} L / \rho_{b}$ (dotdashed line) and the solution $\theta_{b}^{\text {late }}$ (dotted line) are also plotted: note how the numerics agree with $\theta_{b}^{\text {late }}$ at late times. The cosmological parameters of the flat $\Lambda C D M$ model are $\Omega_{b} h^{2}=0.022, \Omega_{c} h^{2}=0.123, \tau=0.04, n_{s}=1, H_{0}=$ $72 \mathrm{~km} \mathrm{~s}^{-1} \mathrm{Mpc}^{-1}$.
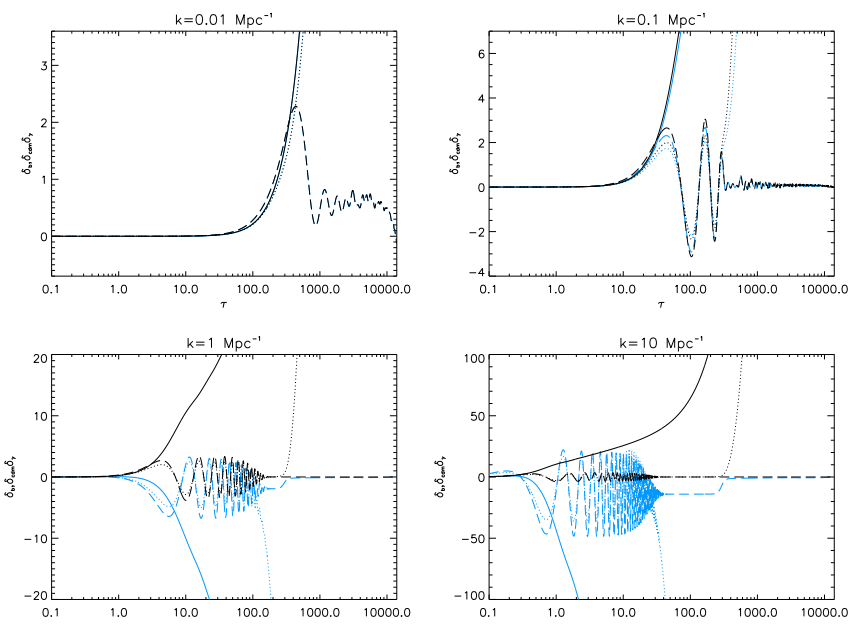

FIG. 7: Evolution of baryons (dotted), CDM (solid) and photons (dashed) density contrast for 4 different wavenumbers with fully correlated (blue) and without (black) PMF. The cosmological parameters are the same of Fig. (6).

density contrasts and therefore the CMB APS in an intermediate range of multipoles before high $\ell$ increase.

\section{RESULTS FOR THE MATTER POWER SPECTRA}

In Fig. (13) we present the results for the linear CDM power spectrum evaluated at present time in presence of SB of PMF. By analyzing Fourier spectra we have checked that the adiabatic results are recovered for $k>$
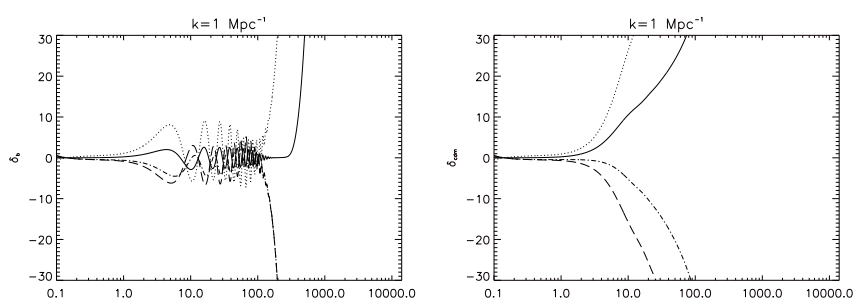

FIG. 8: Time evolution of baryons (left) and CDM (right) density contrasts with vanishing PMF (solid), fully correlated (dashed), fully anti-correlated (dotted) and purely magnetic initial conditions (dot-dashed). The other cosmological parameters are the same as Fig. (6).
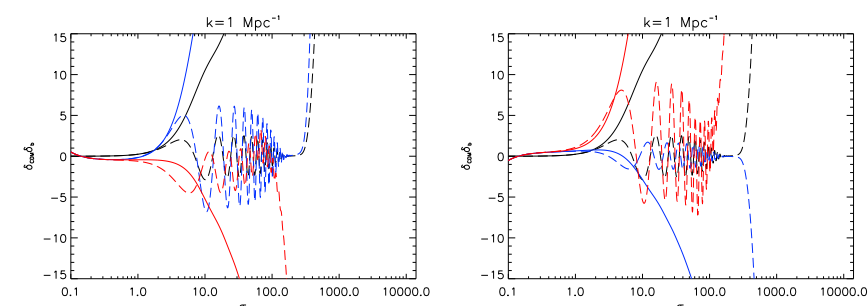

FIG. 9: Time evolution of baryons (dashed) and CDM (solid) density contrasts for purely adiabatic with vanishing PMF (black), fully correlated (left panel) and anti-correlated (right panel) PMF with vanishing (blue) and non vanishing (red) Lorentz force for $k=1 \mathrm{Mpc}^{-1}$. These figures show clearly that the Lorentz force and the gravitational contribution are of opposite sign, and the Lorentz term is more important. The cosmological parameters are the same of Fig. (6).

$2 k_{D}$. We compare the results obtained by neglecting or by taking into account the Lorentz term. By considering the equations evolved and the previous figures, it is clear how the Lorentz term treated as in Eq. (26) is a leading contribution for baryons which gives rise to a long-time effect as show in Fig. 6. Through gravity CDM is affected as shown in Figs. (7-9) and therefore a large feature is present in the linear CDM matter PS.

\section{CONCLUSIONS}

We have investigated the impact of a SB of PMF on scalar cosmological perturbations and its impact on $\mathrm{CMB}$ anisotropies and matter power spectrum. The effects on the CMB angular power spectrum is one of the distinctive features of stochastic PMF together with nongaussianities and Faraday rotation [19]: future missions as PLANCK 20] will greatly improve the present constraints [10, 16].

We have analyzed the SB of PMF in the one-fluid MHD approximation [2] as a source for cosmological perturbations and we have inserted such modifications in the CAMB code [18]. Our numerical code improves previous studies [10, 11] for the treatment of initial conditions 

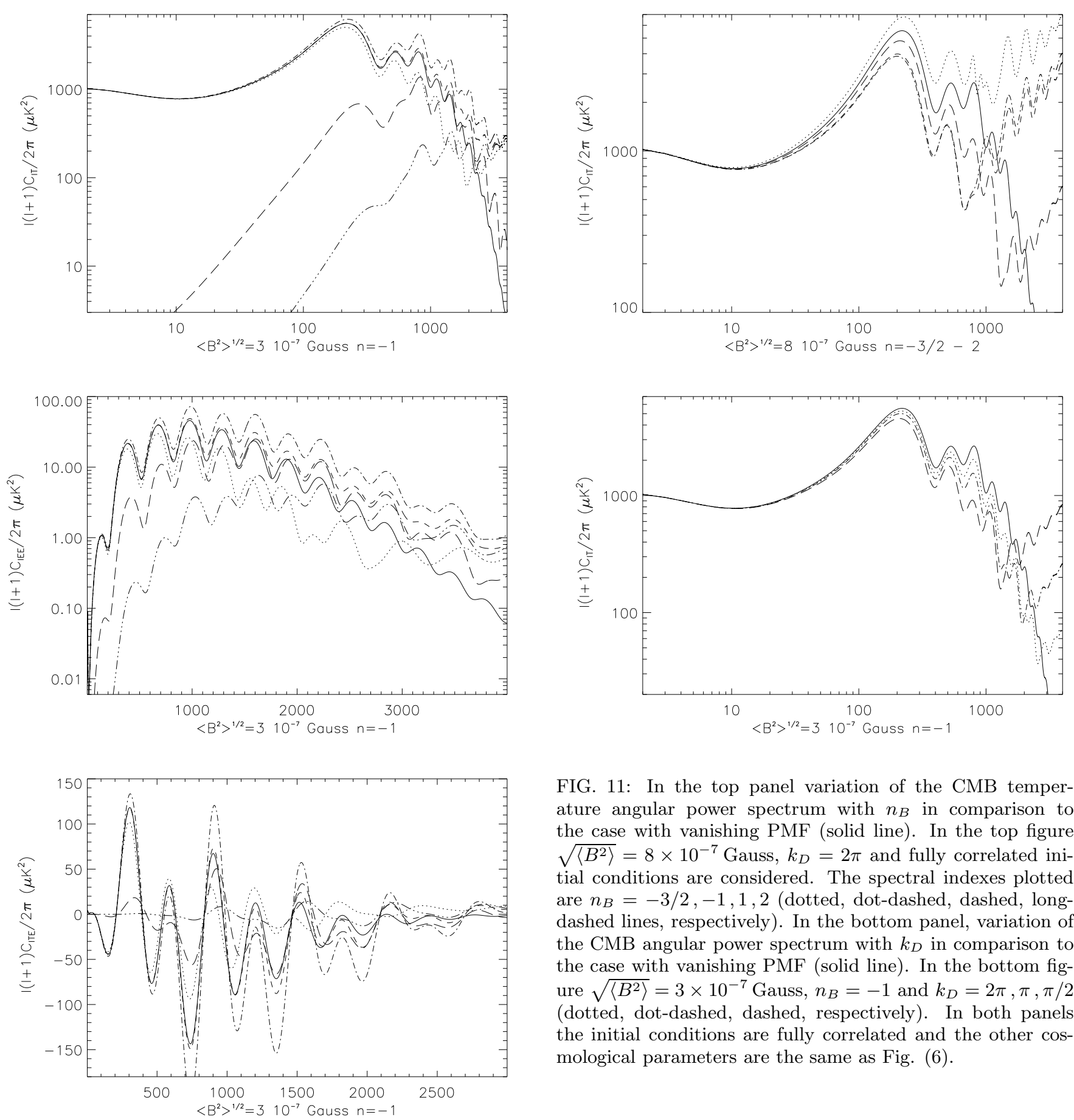

FIG. 11: In the top panel variation of the CMB temperature angular power spectrum with $n_{B}$ in comparison to the case with vanishing PMF (solid line). In the top figure $\sqrt{\left\langle B^{2}\right\rangle}=8 \times 10^{-7}$ Gauss, $k_{D}=2 \pi$ and fully correlated initial conditions are considered. The spectral indexes plotted are $n_{B}=-3 / 2,-1,1,2$ (dotted, dot-dashed, dashed, longdashed lines, respectively). In the bottom panel, variation of the CMB angular power spectrum with $k_{D}$ in comparison to the case with vanishing PMF (solid line). In the bottom figure $\sqrt{\left\langle B^{2}\right\rangle}=3 \times 10^{-7}$ Gauss, $n_{B}=-1$ and $k_{D}=2 \pi, \pi, \pi / 2$ (dotted, dot-dashed, dashed, respectively). In both panels the initial conditions are fully correlated and the other cosmological parameters are the same as Fig. (6).

FIG. 10: CMB temperature angular power spectra obtained with $\sqrt{\left\langle B^{2}\right\rangle}=3 \times 10^{-7}$ Gauss, $n_{B}=-1, k_{D}=\pi$ in comparison with the adiabatic spectrum with vanishing PMF (solid line): TT, EE, TE are displayed in the top, middle, bottom panel, respectively. The purely magnetic, correlation, fully correlated, fully anti-correlated and uncorrelated spectra are represented as triple dotted - dashed, dashed, dotted, dot dashed and long dashed lines, respectively. The other cosmological parameters are the same as Fig. (6).

and exact convolutions for the PMF energy-momentum tensor. Note that the present constraints [10, 16] used neither the correct initial conditions nor the correct convolutions for the PMF energy density and Lorentz force power spectra. Ref. [11] uses the correct initial conditions, but a power spectrum for the PMF energy -density with a spectral index which is twice the one for the power spectrum of the magnetic field. We have shown extensively in Sect. VI and Appendix A,B that this is not the case.

We have also shown how the Lorentz term for baryons 

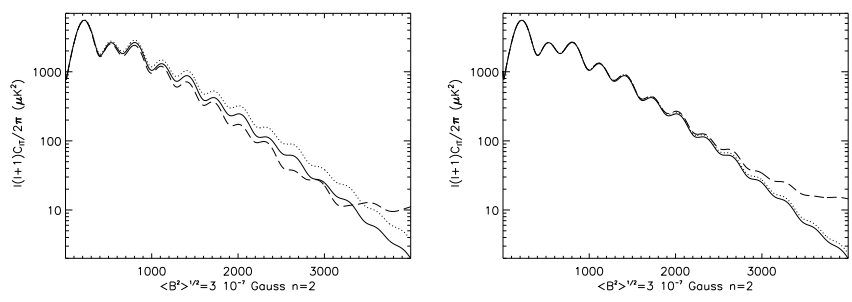

FIG. 12: In the left panel CMB temperature power spectrum obtained with fully correlated PMF with (dashed line) and without (dotted line) Lorentz term in comparison with the vanishing PMF (solid line). As is clear from the previous section, the Lorentz force of a fully correlated magnetic contribution decreases the density contrasts and therefore there is range in which the CMB TT APS is decreased respect to the adiabatic case. In the right panel the same figure with uncorrelated spectra. In the figures $\sqrt{\left\langle B^{2}\right\rangle}=3 \times 10^{-7}$ Gauss, $k_{D}=2 \pi$ and $n_{B}=2$ are considered. The other cosmological parameters are the same as Fig. (6).

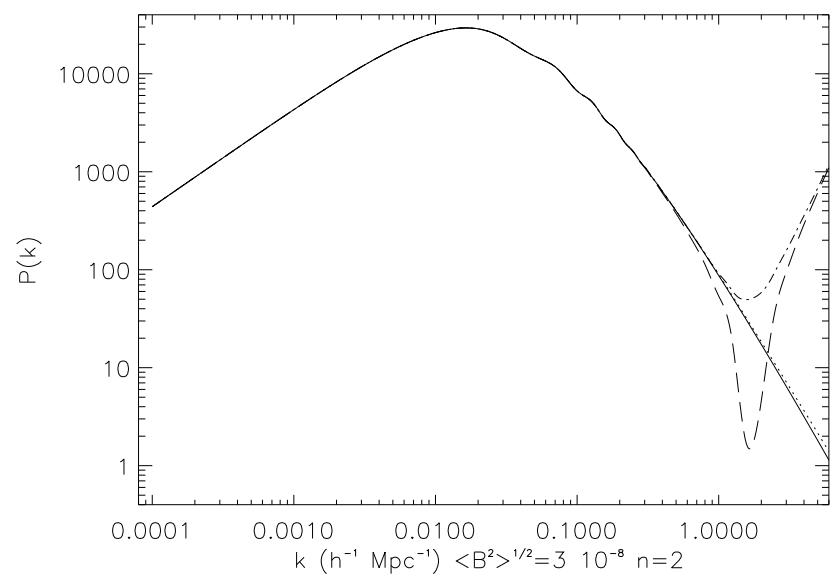

FIG. 13: Linear cold dark matter power spectrum obtained with fully correlated PMF with (dashed line) and without (dotted line) Lorentz term, with uncorrelated PMF and the Lorentz force (dot-dashed line) in comparison with the vanishing PMF (solid line). In the figure $\sqrt{\left\langle B^{2}\right\rangle}=3 \times 10^{-8}$ Gauss, $k_{D}=2 \pi$ and $n_{B}=2$ are considered. The other cosmological parameters are the same as Fig. (6).

in the one-fluid plasma description [2] may lead to a longtime effect which we have described analytically in Eq. (26). This last point deserves further investigation.

\section{Acknowledgments}

We are grateful to Chiara Caprini, Ruth Durrer and Jose Alberto Rubino-Martin for conversations and discussions on magnetic fields. This work has been done in the framework of the Planck LFI activities and is partially supported by ASI contract Planck LFI Activity of Phase E2. We thank INFN IS PD51 for partial support. F. F. is partially supported by INFN IS BO 11.

\section{NOTE ADDED}

While this paper was about to be completed, an article 21] which computes numerically the convolution integrals by taking into account the angular part appeared. Ref. 21] does not display the dependence on $k$ of these convolution integrals and therefore we cannot compare our analytical expressions with their results.

\section{APPENDIX A: ENERGY DENSITY}

The convolution which gives the magnetic energy density spectrum is given in Eq. (16) with the parametrization for the PS for the magnetic field given in Eq. (17). Let us compute the energy density of magnetic field applying the sharp cut-off to both spectra in the convolution according to Eq. (VA). In this case we have two conditions of existence to take into account:

$$
p<k_{D}, \quad|\vec{k}-\vec{p}|<k_{D}
$$

This second condition poses a $k$-dependence on the angular integration domain and, together with the first one, allows the energy power spectrum to be defined for $0<k<2 k_{D}$. For simplicity of notation we normalize the Fourier wavenumber to $k_{D}$ and perform the integration with this choice. The double integral (over $\gamma$ and over $p$ ) we have to compute must therefore be splitted in three parts depending on the $\gamma$ and $p$ lower and upper $k$-depending bounds. This splitting is well displayed in $(k, p)$ plane as showed in Fig.(14): in region $a$ the angular integration has to be done between -1 and 1 , while within $b$ and $c$ regions between $\left(k^{2}+p^{2}-1\right) / 2 k p$ and 1 . 
A sketch of the integration is thus the following:

1) $0<k<1$

$$
\int_{0}^{1-k} d p \int_{-1}^{1} d \gamma \cdots+\int_{1-k}^{1} d p \int_{\frac{k^{2}+p^{2}-1}{2 k p}}^{1} d \gamma \cdots \equiv \int_{0}^{1-k} d p I_{a}(p, k)+\int_{1-k}^{1} d p I_{b}(p, k)
$$

2) $1<k<2$

$$
\int_{k-1}^{1} d p \int_{\frac{k^{2}+p^{2}-1}{2 k p}}^{1} d \gamma \cdots \equiv \int_{k-1}^{1} d p I_{c}(p, k)
$$

The angular integrals can be performed as:

$$
\begin{aligned}
I_{a}= & \int_{-1}^{1} p^{n+2}\left[2-\frac{k^{2}\left(1-\gamma^{2}\right)}{k^{2}+p^{2}-2 k p \gamma}\right]\left(k^{2}+p^{2}-2 k p \gamma\right)^{n / 2} d \gamma \\
= & \frac{2 p^{n-1}}{k n(2+n)(4+n)}\left[(k+p)^{n+2}\left(k^{2}-k(2+n) p+\left(1+4 n+n^{2}\right) p^{2}\right)\right. \\
& \left.-|k-p|^{n+2}\left(k^{2}+k(2+n) p+\left(1+4 n+n^{2}\right) p^{2}\right)\right], \\
I_{b}=I_{c}= & \int_{k^{2}+p^{2}-1}^{2 k p} p^{n+2}\left[2-\frac{k^{2}\left(1-\gamma^{2}\right)}{k^{2}+p^{2}-2 k p \gamma}\right]\left(k^{2}+p^{2}-2 k p \gamma\right)^{n / 2} d \gamma \\
= & \frac{p^{n-1}}{4 k n(2+n)(4+n)}\left[8 k^{4}+2 n-8 k^{2} n+6 k^{4} n+n^{2}-2 k^{2} n^{2}+k^{4} n^{2}\right. \\
& -16 k^{2} p^{2}+24 n p^{2}-12 k^{2} n p^{2}+6 n^{2} p^{2}-2 k^{2} n^{2} p^{2}+8 p^{4}+6 n p^{4}+n^{2} p^{4} \\
& \left.-8|k-p|^{n+2}\left(k^{2}+k(2+n) p+\left(1+4 n+n^{2}\right) p^{2}\right)\right]
\end{aligned}
$$

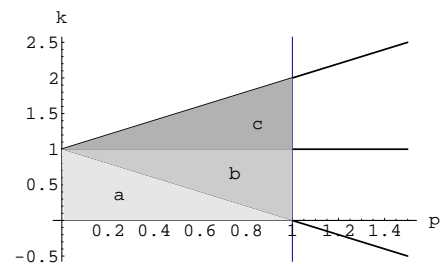

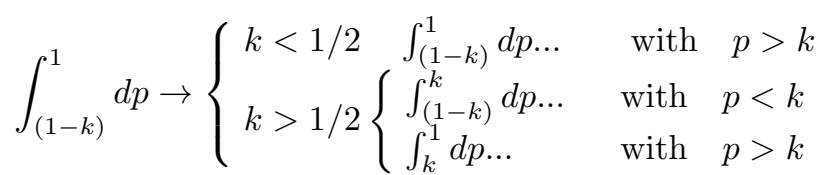

FIG. 14: Integration domains in $(k, p)$ plane

Note that the divergent terms at the denominator $n$ and $n+2$ simply means that the above formulae are not applicable for $n=0$ and $n=-2$ (logarithmic terms appear in these cases).

A special care must be taken in the radial integral.In particular the presence of the term $|k-p|^{n+2}$ in both integrands, when considering odd spectral indexes, makes necessary to divide the two cases: $p<k$ and $p>k$.This leads to a further division of the integration domain. The scheme of the radial integration is then:

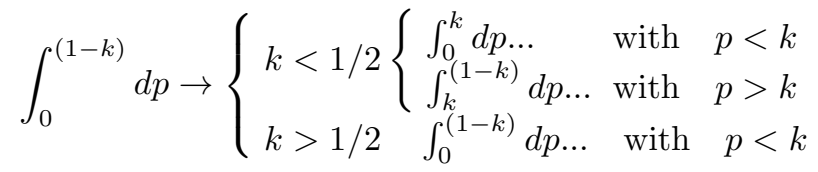

$$
\int_{(k-1)}^{1} d p \rightarrow\left\{1<k<2 \quad \int_{(k-1)}^{1} d p \ldots \quad \text { with } \quad p<k\right.
$$

It is important to study some relevant behaviour of the integrands in $p$. For $p \sim 0$ :

$$
I_{a} \sim \frac{8}{3} k^{n} p^{n+2},
$$


For $p \sim k$ the above integrands behave as

$$
\begin{gathered}
I_{a} \sim \frac{2 k^{n-2}}{n(n+2)(n+4)}\left[2^{n+2} k^{n+4} n(n+3)-\left((k-p)^{2}\right)^{\frac{n+2}{2}}(n+1)(n+4) k^{2}\right], \\
I_{b} \sim \frac{k^{n-2}}{4 n(n+2)(n+4)}\left[n\left(4(n+4) k^{2}+n+2\right)-8\left((k-p)^{2}\right)^{\frac{n+2}{2}}(n+1)(n+4) k^{2}\right] .
\end{gathered}
$$

For $I_{c}, p \sim k$ cannot be obtained since $1<k<2$. It is important to stress that for $n>-3$ the divergences in $p \sim 0$ and $p \sim k$ are integrable. The coefficients of both leading terms are proportional to $k^{n}$.

Following the scheme (A2) we can perform the integration over $p$. Our exact results are given for particular values of $n_{B}$.

1. $n_{B}=4$

$$
\begin{aligned}
& \left|\rho_{B}(k)\right|_{n_{B}=4}^{2}=\frac{A^{2} k_{D}^{11}}{64 \pi k_{*}^{8}}\left[\frac{4}{11}-\tilde{k}+\frac{4}{3} \tilde{k}^{2}-\tilde{k}^{3}+\frac{8}{21} \tilde{k}^{4}-\frac{\tilde{k}^{5}}{24}-\frac{\tilde{k}^{7}}{192}+\frac{\tilde{k}^{11}}{9856}\right] \\
& \text { 2. } n_{B}=3 \\
& \left|\rho_{B}(k)\right|_{n_{B}=3}^{2}=\frac{A^{2} k_{D}^{9}}{64 \pi k_{*}^{6}} \begin{cases}\frac{4}{9}-\tilde{k}+\frac{20}{21} \tilde{k}^{2}-\frac{5}{12} \tilde{k}^{3}+\frac{4}{75} \tilde{k}^{4}+\frac{4}{315} \tilde{k}^{6}-\frac{\tilde{k}^{9}}{525} & \text { for } \quad 0 \leq \tilde{k} \leq 1 \\
(2-\tilde{k})^{2} \frac{264-436 \tilde{k}+863 \tilde{k}^{2}-528 \tilde{k}^{3}+48 \tilde{k}^{5}+48 \tilde{k}^{6}+16 \tilde{k}^{7}+4 \tilde{k}^{8}}{6300 k} & \text { for } \quad 1 \leq \tilde{k} \leq 2\end{cases}
\end{aligned}
$$$$
\text { 3. } n_{B}=2
$$$$
\left|\rho_{B}(k)\right|_{n_{B}=2}^{2}=\frac{A^{2} k_{D}^{7}}{64 \pi k_{*}^{4}}\left[\frac{4}{7}-\tilde{k}+\frac{8}{15} \tilde{k}^{2}-\frac{\tilde{k}^{5}}{24}+\frac{11}{2240} \tilde{k}^{7}\right]
$$

4. $n_{B}=1$

$$
\left|\rho_{B}(k)\right|_{n_{B}=1}^{2}=\frac{A^{2} k_{D}^{5}}{64 \pi k_{*}^{2}} \begin{cases}\frac{4}{5}-\tilde{k}+\frac{1}{4} \tilde{k}^{3}+\frac{4}{15} \tilde{k}^{4}-\frac{1}{5} \tilde{k}^{5} & \text { for } \quad 0 \leq \tilde{k} \leq 1 \\ (2-\tilde{k})^{2} \frac{8-4 \tilde{k}-\tilde{k}^{2}+4 \tilde{k}^{4}}{60 k} & \text { for } \quad 1 \leq \tilde{k} \leq 2\end{cases}
$$

5. $n_{B}=0$

$$
\left.\rho_{B}(k)\right|_{n_{B}=0} ^{2}=\frac{A^{2} k_{D}^{3}}{64 \pi}\left\{\begin{array}{l}
\frac{1}{96 \tilde{k}}\left[\tilde{k}\left(116-102 \tilde{k}-84 \tilde{k}^{2}+\tilde{k}^{3}\left(53+4 \pi^{2}\right)\right)+\right. \\
12 \log (1-\tilde{k})\left(-1+4 \tilde{k}^{2}-3 \tilde{k}^{4}+4 \tilde{k}^{4} \log \tilde{k}\right)- \\
\left.48 \tilde{k}^{4} \operatorname{PolyLog}\left[2, \frac{-1+\tilde{k}}{\tilde{k}}\right]\right] \\
\frac{1}{96 \tilde{k}}\left[116 \tilde{k}-102 \tilde{k}^{2}-84 \tilde{k}^{3}+53 \tilde{k}^{4}+\right. \\
\log [-1+\tilde{k}]\left(-12+48 \tilde{k}^{2}-36 \tilde{k}^{4}+24 \tilde{k}^{4} \log \tilde{k}\right)+ \\
\left.24 \tilde{k}^{4} \operatorname{PolyLog}\left[2, \frac{1}{k}\right]-24 \tilde{k}^{4} \operatorname{PolyLog}\left[2, \frac{-1+\tilde{k}}{\tilde{k}}\right]\right]
\end{array} \quad \text { for } \quad 0 \leq \tilde{k} \leq 1\right.
$$


6. $n_{B}=-1$

$$
\left|\rho_{B}(k)\right|_{n_{B}=-1}^{2}=\frac{A^{2} k_{D} k_{*}^{2}}{64 \pi} \begin{cases}4-5 \tilde{k}+\frac{4 \tilde{k}^{2}}{3}+\frac{\tilde{k}^{3}}{4} & \text { for } \quad 0 \leq \tilde{k} \leq 1 \\ \frac{\left((-2+\tilde{k})^{2}\left(8-4 \tilde{k}+3 \tilde{k}^{2}\right)\right)}{12 \tilde{k}} & \text { for } \quad 1 \leq \tilde{k} \leq 2\end{cases}
$$

7. $n_{B}=-3 / 2$

$$
\left|\rho_{B}(k)\right|_{n=-3 / 2}^{2}=\frac{A^{2} k_{*}^{3}}{64 \pi}\left\{\begin{array}{l}
\frac{1}{45}\left[\frac{8\left(-33+29 \tilde{k}-4 \tilde{k}^{2}+8 \tilde{k}^{3}\right)}{\sqrt{1-\tilde{k} \tilde{k}}+\frac{264}{\tilde{k}}+60 \tilde{k}+5 \tilde{k}^{3}}\right. \\
-90 \pi+360 \log [1+\sqrt{1-k}]-180 \log \tilde{k}] \\
\frac{1}{45}\left[-\frac{\left(8\left(-33+29 \tilde{k}-4 \tilde{k}^{2}+8 \tilde{k}^{3}\right)\right.}{\sqrt{-1+\tilde{k} \tilde{k}}}+\frac{264}{\tilde{k}}+60 k+5 k^{3}\right. \\
\left.-180 \arctan \left[\frac{1}{\sqrt{-1+\tilde{k}}}\right]+180 \arctan [\sqrt{-1+\tilde{k}}]\right] \quad \text { for } \quad 1 \leq \tilde{k} \leq 2
\end{array}\right.
$$

\section{APPENDIX B: LORENTZ FORCE}

In order to obtain the complete estimate of the contribution of PMFs to the perturbation evolution is necessary to solve the convolution for the Lorentz Force power spectrum. The anisotropic stress can be obtained directly from its relation with the Lorentz force and the magnetic energy density. The convolution which gives the Lorentz force is given in Eq. (23) with the parametrization for the PS for the magnetic field given in Eq. (17).

$$
\begin{aligned}
& \text { 1. } n_{B}=4 \\
& |L(k)|_{n_{B}=4}^{2}=\frac{A^{2} k_{D}^{11}}{64 \pi k_{*}^{8}} \begin{cases}\frac{4}{15}-\frac{16 \tilde{k}}{15}+\frac{317 \tilde{k}^{2}}{135}-\frac{10 \tilde{k}^{3}}{3}+\frac{45 \tilde{k}^{4}}{14}-\frac{1481 \tilde{k}^{5}}{720}+\frac{4 \tilde{k}^{6}}{5}-\frac{977 \tilde{k}^{7}}{6720}+\frac{1357 \tilde{k}^{11}}{2661120} & \text { for } \quad 0 \leq \tilde{k} \leq 1 \\
\frac{248}{1155}-\frac{2 \tilde{k}}{3}+\frac{137 \tilde{k}^{2}}{135}-\frac{5 \tilde{k}^{3}}{6}+\frac{5 k^{4}}{14}-\frac{41 \tilde{k}^{5}}{720}-\frac{17 \tilde{k}^{7}}{6720}+\frac{41 k^{11}}{532224} & \text { for } \quad 1 \leq \tilde{k} \leq 2\end{cases} \\
& \text { 2. } n_{B}=3
\end{aligned}
$$

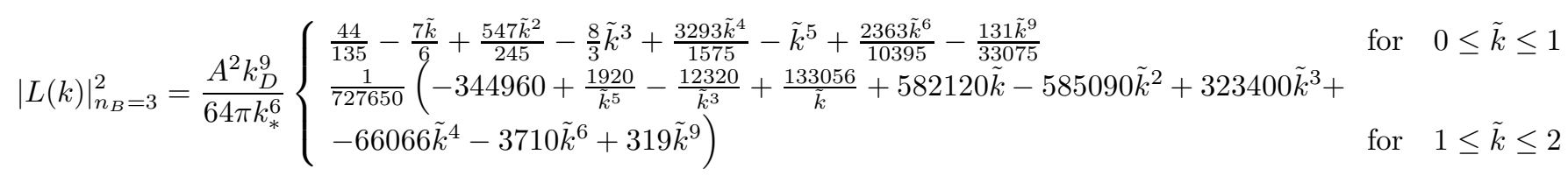$$
\text { 3. } n_{B}=2
$$$$
|L(k)|_{n_{B}=2}^{2}=\frac{A^{2} k_{D}^{7}}{64 \pi k_{*}^{4}} \begin{cases}\frac{44}{105}-\frac{4 \tilde{k}}{3}+\frac{11 \tilde{k}^{2}}{5}-\frac{13 \tilde{k}^{3}}{6}+\frac{4 \tilde{k}^{4}}{3}-\frac{33 \tilde{k}^{5}}{80}+\frac{7 \tilde{k}^{7}}{320} & \text { for } \quad 0 \leq \tilde{k} \leq 1 \\ \frac{32}{105}-\frac{2 k}{3}+\frac{3 \tilde{k}^{2}}{5}-\frac{\tilde{k}^{3}}{6}-\frac{\tilde{k}^{5}}{80}+\frac{19 \tilde{k}^{7}}{6720} & \text { for } \quad 1 \leq \tilde{k} \leq 2\end{cases}
$$ 
4. $n_{B}=1$

$$
|L(k)|_{n=1}^{2}=\frac{A^{2} k_{D}^{5}}{64 \pi k_{*}^{2}} \begin{cases}\frac{44}{75}-\frac{5 \tilde{k}}{3}+\frac{761 \tilde{k}^{2}}{315}-2 \tilde{k}^{3}+\frac{659 \tilde{k}^{4}}{630}-\frac{41 \tilde{k}^{5}}{150} & \text { for } \quad 0 \leq \tilde{k} \leq 1 \\ \frac{128-480 k^{2}+2240 k^{4}-4368 k^{5}+4200 k^{6}-1310 k^{7}-145 k^{9}+77 k^{10}}{3150 k^{5}} & \text { for } \quad 1 \leq \tilde{k} \leq 2\end{cases}
$$

5. $n_{B}=0$

$$
|L(k)|_{n_{B}=0}^{2}=\frac{A^{2} k_{D}^{3}}{64 \pi} \begin{cases}\frac{1}{1152 \tilde{k}^{5}}\left[12\left(-1+\tilde{k}^{2}\right)^{3}\left(3+\tilde{k}^{2}\right) \log [1-\tilde{k}]+\right. \\ 12\left(-3+8 \tilde{k}^{2}-30 \tilde{k}^{4}+64 \tilde{k}^{5}-48 \tilde{k}^{6}+9 \tilde{k}^{8}\right) \log [1-\tilde{k}]+ & \text { for } \quad 0 \leq \tilde{k} \leq 1 \\ \left.\tilde{k}\left(-72-36 \tilde{k}+168 \tilde{k}^{2}+78 \tilde{k}^{3}+744 \tilde{k}^{4}-2484 \tilde{k}^{5}+4728 \tilde{k}^{6}-2869 \tilde{k}^{7}+1536 \tilde{k}^{7} \log \tilde{k}\right)\right] & \frac{1}{1152 k^{5}}[(2-k) k(-36+k(-36+k(66+k(72+k(152+k(-14+53 k)))))+ \\ \left.24\left(-3+k^{2}\left(8+k^{2}\left(-18+k\left(32-24 k+5 k^{3}\right)\right)\right)\right) \log (-1+\tilde{k})\right] & \text { for } \quad 1 \leq \tilde{k} \leq 2\end{cases}
$$

6. $n_{B}=-1$

$$
|L(k)|_{n_{B}=-1}^{2}=\frac{A^{2} k_{D} k_{*}^{2}}{64 \pi} \begin{cases}\frac{44}{15}-\frac{83 \tilde{k}^{2}}{35}+4 \tilde{k} \log \tilde{k} & \text { for } \quad 0 \leq \tilde{k} \leq 1 \\ \frac{-64+\tilde{k}^{2}\left(112+\tilde{k}^{3}(112+\tilde{k}(-140+39 \tilde{k}))\right)}{105 \tilde{k}^{5}} ; & \text { for } \quad 1 \leq \tilde{k} \leq 2\end{cases}
$$

7. $n_{B}=-3 / 2$

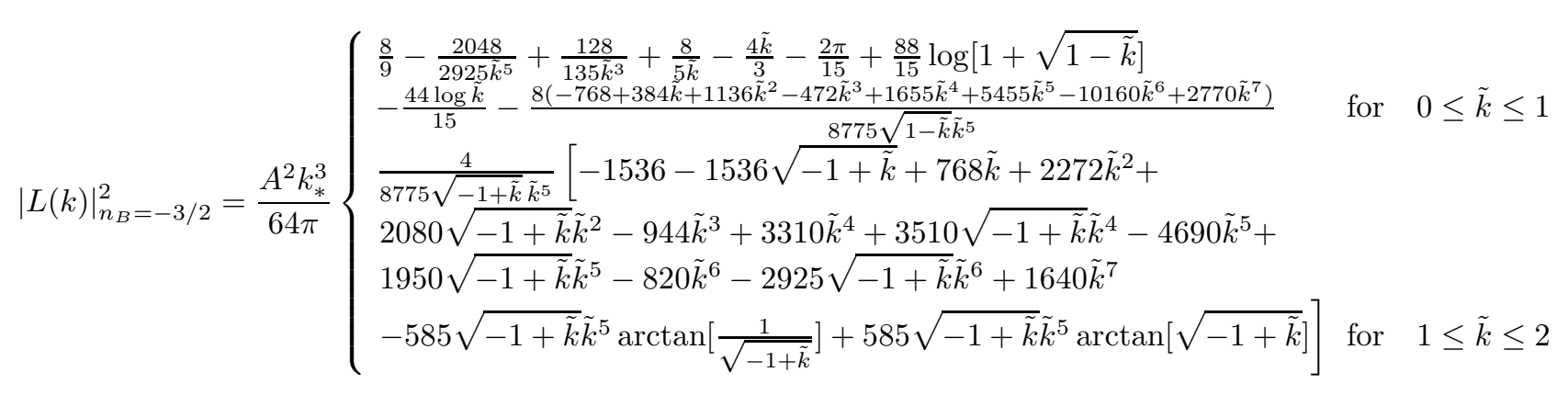

[1] D. Grasso and H. R. Rubinstein, Phys. Rept. 348 (2001) 163

[2] M. Giovannini, Class. Quant. Grav. 23 (2006) R1.

[3] K. Subramanian, "Primordial magnetic fields and CMB anisotropies," arXiv:astro-ph/0601570

[4] A. Mack, T. Kahniashvili and A. Kosowsky, Phys. Rev. D 65 (2002) 123004

[5] A. Lewis, Phys. Rev. D 70 (2000) 043011

[6] R. Durrer, P. G. Ferreira and T. Kahniashvili, Phys. Rev. D 61 (2000) 043001

[7] C. Caprini, R. Durrer and T. Kahniashvili, Phys. Rev. D 69 (2004) 063006
[8] T. Kahniashvili and B. Ratra, Phys. Rev. D 75, 023002 (2006)

[9] S. Koh, \& C. H. Lee, Phys. Rev. D 62, (2000) 083509.

[10] D. G. Yamazaki, K. Ichiki, T. Kajino, and G. J. Mathews, Astrophys. J. , 646, 719 (2006).

[11] M. Giovannini and K. E. Kunze, arXiv:0712.3483 [astro$\mathrm{ph}]$.

[12] J. A. Adams, U. H. Danielsson, D. Grasso and H. Rubinstein, Phys. Lett. B 388, (1996) 253

[13] C. P. Ma and E. Bertschinger, Astrophys. J. , 455 (1995) 7.

[14] M. Giovannini, Phys. Rev. D 74 (2006) 063002 
[15] D. Paoletti, Einstein-Boltzmann Codes for Cosmic Microwave Background Anisotropies with Primordial Magnetic Fields, Tesi di Laurea Specialistica in Astrofisica e Cosmologia, March 2007, Universitá degli Studi di Bologna (unpublished).

[16] D. G. Yamazaki, K. Ichiki and T. Kajino, Astrophys. J. 625 (2005) L1

[17] M. Abramowitz and I. Stegun, Handbook of mathematical functions with formulas, graphs, and mathematical table, New York: Dover Publishing (1965).
[18] A. Lewis, A. Challinor and A. Lasenby, Astrophys. J. 538 (2000) 473

[19] A. Kosowsky, T. Kahniashvili, G. Lavrelashvili and B. Ratra, Phys. Rev. D 71 (2005) 043006

[20] [Planck Collaboration], "Planck: The scientific programme," arXiv:astro-ph/0604069 (2006).

[21] D. G. Yamazaki, K. Ichiki, T. Kajino and G. J. Mathews, Phys. Rev. D 77 (2008) 043005 